\title{
Sexo, estilos cognitivos e mapas cognitivos: avaliando suas relações na população geral
}

\author{
Taciano Lemos Milfont \\ Victoria University of Wellington \\ Jorge Artur Peçanha de Miranda Coelho \\ Universidade Federal de Alagoas \\ Viviany Silva Pessoa \\ Valdiney Veloso Gouveia \\ Universidade Federal da Paraíba
}

\begin{abstract}
Resumo
Este artigo relata em que medida homens e mulheres se diferenciam quanto ao estilo cognitivo de sistematização e à habilidade de construção de mapas cognitivos. Participaram da pesquisa 214 pessoas $(57 \%$ mulheres) de João Pessoa (PB), com idade média de 29,3 anos. Elas foram requeridas a listar as Unidades da Federação (UFs) e capitais brasileiras, indicar em mapa esquemático as UFs do país, estimar as distâncias entre capitais e responder o quociente de sistematização. Os resultados indicaram que homens e mulheres se diferenciam em relação ao número de acertos entre capitais e à pontuação em sistematização; em média, os homens apresentam mais acertos e são mais sistematizadores. Em relação à estimativa de distâncias, verificou-se uma configuração espacial mais adequada para as pessoas do sexo masculino. Concluindo, o sexo e o estilo cognitivo de sistematização influenciam a construção de mapas cognitivos.
\end{abstract}

Palavras-chave: mapas cognitivos; estilos cognitivos; sistematização; sexo.

\begin{abstract}
Gender, systemizing style and cognitive maps: examining their links in the general population. This study examined the extent to which men and women differ regarding systemizing and construction of cognitive maps. Participants were 214 people (57\% women) from João Pessoa (Brazil), with mean age of 29.3 years. They were asked to list the Brazilian Federation Unities (BFUs) and their capitals, to indicate in a sketch map the BFUs, to estimate the distances between capitals, and to answer the systemizing quotient test and demographic questions. Results showed that men and women differ with respect to the number of BFUs-capital correctly listed, and on the systemizing score, with men showing more right answers and being more systemizing than women. Men and women did not differ in locating the BFUs in the map, but men had more correct answers overall. In terms of distance estimation once again men showed better spatial representation than women. These findings indicate the influence of sex and systematizing on the representation of cognitive maps.
\end{abstract}

Keywords: cognitive maps; cognitive styles; systemizing; sex.

$\mathrm{O}$ uso de mapas para orientação no ambiente é antigo na história da humanidade. Os antepassados nômades, em busca de locais que lhes oferecessem melhores condições para manutenção da vida, utilizavam desenhos de vales e rios nas paredes das grutas como referencial para orientação no ambiente (Gomes \& Pinheiro, 1998).

Não por acaso, psicólogos e geógrafos há muito têm se dedicado ao estudo da natureza e do processo de uso de mapas cognitivos; especificamente, interessam-se em conhecer como as pessoas representam mentalmente o ambiente. Neste particular, um aspecto que chama a atenção são as diferenças de habilidades decorrentes do sexo, com homens e mulheres presumivelmente diferindo no uso e representação de mapas cognitivos.

As especulações sobre estas diferenças de sexo podem ser estabelecidas a partir de teorias biológicas hormonais, lateralidade cerebral, estratégias evolutivas, aprendizagem e socialização, feedback parental e societal (Montello, Lovelace, Golledge, \& Self, 1999). A suposição de que as diferenças entre sexos são produzidas pelo meio sociocultural no qual estão inseridos homens e mulheres sustenta que os ambientes produzem e reforçam estilos comportamentais diferenciados e há uma tendência cultural em agrupar meninos e meninas em ambientes distintos.

Neste sentido, os papéis sociais comumente assumidos por 
meninos e meninas seriam os determinantes destas diferenças. Os meninos são tradicionalmente incentivados a atividades que permitem explorar mais o ambiente (por exemplo, jogar bola na vizinhança, acompanhar o pai em atividades de pesca e esportes), enquanto as meninas ficam "protegidas" em casa, realizando atividades relacionadas com o cuidado e a vida doméstica (por exemplo, brincar com bonecas, ajudar a mãe em tarefas do lar). Portanto, o contato com o mundo e a experiência de orientação espacial das meninas são menos evidentes quando comparados com as possibilidades de exploração vivenciadas pelos meninos. Assim, o desenvolvimento é influenciado por estas diferenças em socialização, sendo que meninos e meninas se tornam homens e mulheres que, em princípio, guardam tais diferenças em comportamentos que refletem suas habilidades em lidar com o ambiente (Gomes \& Pinheiro, 1998).

Embora tenha implicação em comportamentos diversos, o sexo é uma variável de natureza estrutural. Não obstante, estima-se que possa também ser representada por estilos psicológicos ou cognitivos, a exemplo das dimensões de empatia e sistematização (Baron-Cohen, 2002). Ainda que detalhadas a seguir, cabe definir estas dimensões brevemente aqui.

A empatia é definida como a habilidade para identificar estados mentais alheios e responder a estes de forma emocionalmente apropriada, sendo apresentada em maior magnitude por pessoas do sexo feminino (Gouveia, Milfont, Gouveia, Rique, \& Galvão, no prelo).

A sistematização compreende a habilidade para analisar um sistema, tomando como referência regras de causa e consequência, sendo mais característica em homens (Billington, Baron-Cohen, \& Wheelwright, 2007; Focquaert, Steven, Wolford, Colden, \& Gazzaniga, 2007). Baron-Cohen (2002) sugere que seja biológica a origem das diferenças relacionadas ao sexo em empatia e sistematização, compreendendo parte da condição de ser humano e não produto da experiência sociocultural do indivíduo.

A propósito, Baron-Cohen (2002) destaca resultados de pesquisa experimental que demonstram o maior interesse (tempo de observação) de bebês do sexo masculino por móbile (objeto que envolve regras de movimento) do que pela face humana (não envolve regras de movimento, mas a expressão de emoções). Por outro lado, as meninas apresentam maior interesse pela face humana do que por móbile. Estas diferenças de sexo são, portanto, apresentadas muito cedo, sem a experiência e exposição à cultura propriamente dita, tal como nas fases posteriores da vida. Isso sugere que, apesar de a cultura e a socialização determinarem em parte as diferenças sexuais, a biologia pode igualmente contribuir para estas diferenças em termos do "tipo de cérebro" masculino (maior interesse por sistemas) e feminino (maior interesse por empatia).

Considerando o interesse em compreender diferenças pessoais em mapas cognitivos, o presente estudo tem em conta duas variáveis específicas: o sexo e o estilo cognitivo de sistematização. Estas não são tratadas como processo de naturalização de estereotipias de diferenças sexuais, mas em função de achados empíricos que demonstram a relação entre sexo, estilo cognitivo e habilidade espacial.

Levando em conta a escassez de dados sobre mapas cognitivos, estilos cognitivos e sexo no contexto brasileiro (Gomes \& Pinheiro, 1998; Pinheiro, 1998), buscou-se conhecer diferenças e semelhanças entre homens e mulheres quanto ao estilo cognitivo de sistematização e à habilidade de construção do mapa cognitivo do Brasil, considerando respostas verbais e visuo-espaciais. Procura-se a seguir descrever os principais construtos de interesse (mapas e estilos cognitivos), passando posteriormente a considerar as diferenças de sexo para explicar as habilidades referentes aos mapas cognitivos.

\section{Mapas cognitivos}

Mapas cognitivos podem ser concebidos como um construto que engloba processos psicológicos que possibilitam ao indivíduo registrar, codificar, armazenar, recordar e manipular a informação sobre seu ambiente espacial (Downs \& Stea, 1973). Portanto, a cognição espacial inclui a noção de mapas cognitivos; isto é, imagens pictóricas e semânticas de como os lugares estão organizados (Kitchin, 1994).

Os mapas cognitivos são representações internas de como se conhece o mundo e o ambiente em que se está inserido. No processo de tomada de decisão, tais mapas são acessados para prover informações relativas à situação ou tarefa que se impõe, como, por exemplo, a localização no ambiente. Tem implicação neste processo o ambiente em que a decisão deve ser aplicada e os comportamentos exigidos em função da decisão ou escolha tomada (Golledge, 2002).

É também possível compreender os mapas cognitivos em relação às localizações percebidas das pessoas e objetos no ambiente, o que corresponde a imagens análogas a mapas (tais como mapas geográficos), que as pessoas utilizam para navegar no ambiente (espaço natural ou construído). Estas cognições e memórias de lugares produzem imagens mentais que possibilitam ao indivíduo ter uma representação mental organizada de seu ambiente (por exemplo, país, cidade, bairro, casa, ou outros lugares específicos). Sua função é facilitar a apreensão dos ambientes e a tomada de decisões espaciais no que concerne à localização e ao movimento no ambiente (Aragonés, 1991; Gomes \& Pinheiro, 1998; Kitchin, 1994; Moser \& Uzzell, 2003). É importante salientar que tais mapas têm três componentes fundamentais de informação, a saber: tamanho, distância e direção do espaço, a partir dos quais as pessoas tomam as decisões necessárias para ir de um lugar a outro (Aragonés, 1991).

De fato, todo mapa cognitivo constitui um processo mental de elaboração e armazenamento de informações que possibilitam simplificar, codificar e ordenar o infindável e complexo mundo em que as pessoas estão inseridas: o ambiente. Mas, como este processo ocorre? Existem diferenças sexuais neste processo? Tais questionamentos podem estar relacionados com o estilo cognitivo de sistematização, descritos a seguir.

\section{Estilos cognitivos}

Como comentado acima, Baron-Cohen (2002) propõe duas dimensões psicológicas (Empathizing-Systemizing Theory or E-S Theory) ou estilos cognitivos que diferem quanto aos sexos: Empatia e Sistematização. Este último diz respeito à habilidade para compreender e predizer as leis que governam o universo 
inanimado, enquanto empatia representa a habilidade para compreender e predizer o mundo social (Baron-Cohen, Richler, Bisarya, Gurunathan, \& Wheelwright, 2003).

A empatia permite ao indivíduo prever o comportamento e ter cuidado e preocupação com os sentimentos de outros; facilita a interação no mundo social e é definida como uma habilidade para identificar estados mentais, incluindo emoções de outros e responder em conformidade. Esta se constitui de componentes cognitivo e afetivo. O componente cognitivo envolve o entendimento dos pensamentos e sentimentos dos outros, enquanto o afetivo diz respeito a respostas emocionais que surgem como resultado da compreensão do estado emocional de outros (Baron-Cohen, 2002; Baron-Cohen \& cols., 2003; Baron-Cohen \& Wheelwright, 2004; Lawrence \& cols., 2004; Wheelwright \& cols., 2006). Este estilo é apresentado em maior medida por pessoas do sexo feminino (Baron-Cohen, 2002; Billington et al., 2007; Focquaert, Steven, Wolford, Colden, \& Gazzaniga, 2007; Nettle, 2007; Wheelwright \& cols., 2006).

O estilo cognitivo de sistematização, por outro lado, expressa uma tendência a analisar as regras subjacentes a um sistema. Baron-Cohen (2002) chama a atenção para o que se entende por sistema em sua proposição teórica. Sumarizando, ele o concebe como qualquer coisa que tenha entrada e saída (input e output). Ao sistematizar, utiliza-se como regra a proposição "se x, então y". O cérebro concentra-se em detalhes e parâmetros do sistema, isto é, a entrada e saída e respectivas variações ("se eu fizer $x$, em seguida acontece y"). Refere-se ainda à unidade base para construção de sistemas, que são encontrados em diferentes domínios: técnico (máquinas e ferramentas), natural (sistema meteorológico, clima), abstrato (matemática), social (sistema político), espacial (leitura de mapas) e organização (taxonomia).

Apesar dos vários domínios, todos os sistemas compartilham de uma estrutura tripartida: todos podem ser analisados em termos do princípio: entrada-operação-saída (input-operation-output). A entrada (input) é definida como o estado inicial do sistema; a saída (output) é o subsequente estado do sistema; e a operação (operation) é definida como ações que transformam a entrada em saída. Contudo, a sistematização é um processo indutivo. $\mathrm{O}$ indivíduo analisa o que acontece, recolhe informações, muitas vezes quantifica as diferenças das variáveis do sistema e sua relação com variações de resultados. Após a confirmação de um padrão confiável de associação e resultados previsíveis, formase uma regra sobre o funcionamento do sistema. A menos que ocorra uma exceção, os procedimentos são refinados ou revistos; caso contrário, a regra é mantida. Evidências indicam que os homens, em média, são mais sistemáticos do que as mulheres (Baron-Cohen, 2002; Baron-Cohen \& cols., 2003; Billington et al., 2007; Focquaert \& cols., 2007; Nettle, 2007; Wheelwright $\&$ cols., 2006).

Além de diferenças sexuais, é importante enfatizar que existem também diferenças individuais tanto em empatia como sistematização. De acordo com Baron-Cohen (2002), indivíduos com a empatia mais desenvolvida do que a sistematização são definidos como do Tipo E; aqueles com a sistematização mais desenvolvida do que a empatia são do Tipo $S$; e, finalmente, os indivíduos com a sistematização e a empatia igualmente desenvolvidas são denominados de Tipo $B$ (para indicar o cérebro equilibrado, isto é, balanced brain). Indivíduos cuja sistematização é normal ou hiperdesenvolvida, mas a empatia é hipodesenvolvida, são definidos como Extremo do Tipo S; isto é, são sistematizadores talentosos, mas ao mesmo tempo são incapazes de demonstrar empatia (mind-blind). Por outro lado, existe também o Extremo Tipo E: pessoas que têm empatia normal ou hiperdesenvolvida, porém a sistematização é hipodesenvolvida, sendo incapazes de sistematização (systemblind).

Em média, mais homens do que mulheres têm um cérebro do Tipo $S$, e mais mulheres do que homens têm um cérebro do Tipo E. Os achados sobre a superioridade feminina em empatia indicam melhor capacidade em decifrar comunicação não verbal, perceber nuanças sutis do tom de voz ou expressão facial, e julgar o caráter de uma pessoa. Já os achados sobre a superioridade masculina em sistematização indicam maior domínio de disciplinas como matemática, física e engenharia, que requerem grau elevado de sistematização e maior habilidade espacial (Baron-Cohen, 2002; Baron-Cohen \& cols., 2003; Halpern, 2004). Como os mapas cognitivos necessitam de processos cognitivos, pode-se estimar a influência de sistematização na construção de mapas cognitivos.

\section{Mapas cognitivos e diferenças relacionadas aos sexos}

A literatura indica que homens e mulheres diferem em sua capacidade de habilidades espaciais. Mais especificamente, as pesquisas fundamentadas em dados empíricos levam em conta as diferenças e semelhanças entre homens e mulheres no que concerne à habilidade espacial (Halpern, 2004; Montello \& cols. 1999). Nesta direção, Gomes e Pinheiro (1998) realizaram uma pesquisa com 82 estudantes universitários (46 mulheres e 36 homens) sobre seu desempenho em mapa cognitivo, consistindo no desenho do mapa do mundo feito à mão livre pelo participante. Foram utilizados dois mapas impressos apenas com o contorno do continente, sendo um com o eixo central nas Américas e o outro com o eixo central na Ásia. Os participantes eram solicitados a colocar no mapa todos os países que conseguiam lembrar, considerando o nome, a fronteira (contorno) e a localização, isto é, a combinação de conhecimento verbal e espacial. Constatou-se que homens e mulheres tiveram basicamente o mesmo desempenho quando o fator considerado foi conhecimento verbal; ou seja, os nomes dos países foram guardados na memória e resgatados de modo similar por ambos os grupos. Entretanto, quanto ao 'contorno' e à 'localização' dos países (conhecimento espacial), foi constatado melhor desempenho por parte dos homens. Estes autores concluíram que os homens têm a função espacial mais bem definida do que as mulheres para a representação mental do mapa do mundo. Contudo, chegaram a esta conclusão com base unicamente em comparação de percentuais, sem utilizar testes estatísticos para confirmar as diferenças observadas.

Em outro estudo sobre mapas cognitivos, Pinheiro (1998) contou com 93 estudantes universitários do curso de Geografia (52 mulheres e 41 homens) provenientes de Natal $(n=21)$, Uberlândia $(n=32)$ e Curitiba $(n=40)$. Os participantes foram solicitados a desenhar o mapa do mundo em uma folha em branco, inferindo-se então a construção de mapas cognitivos 
do mundo. As nações mundiais foram avaliadas tanto por sua inclusão no mapa esquemático como por sua ausência. Nenhuma comparação foi feita entre homens e mulheres quanto à habilidade de construção do mapa cognitivo do mundo. A principal influência informada sobre a inclusão ou omissão de nações no mapa esquemático foi de indicadores mundiais quantitativos dos países, como seu poder geopolítico e militar, tamanho e importância econômica, seguidos por semelhanças culturais e localização do país no mapa do mundo.

Montello e cols. (1999), mesmo não tendo tratado especificamente de mapas cognitivos, demonstram diferenças entre homens e mulheres quanto a habilidades espaciais. Em seu estudo com 79 participantes estadunidenses de Santa Barbara (43 mulheres e 36 homens), envolvendo uma bateria de atividades espaciais e geográficas, estes autores reportaram que homens e mulheres diferem em atividades particulares, tendo os homens melhor desempenho naquelas que envolvem elementos métricos e as mulheres nas que consideram elementos verbais. Descrevem que os homens tendem a apresentar melhor desempenho em atividades espaciais que envolvem processos de rotação mental, direção cardinal e estimação de distâncias, enquanto as mulheres tendem a obter melhor desempenho nos processos que implicam conhecimento geográfico verbal; estas também cometeram menos erros em atividades que envolvem processo de memória objeto-localização. Neste sentido, as mulheres tendem a descrever rotas utilizando mais referenciais não métricos (isto é, referenciais verbais) como pontos de referência. Já os homens utilizam mais termos que envolvem descrição de distâncias e direção cardinal, utilizando mais referenciais não verbais (isto é, referenciais métricos) para navegação no ambiente.

A pesquisa de Lawton (2001) parece reforçar a anteriormente descrita. Em levantamento pela Internet sobre referenciais espaciais utilizados para orientação no ambiente, contando com amostra de 240 participantes estadunidenses (154 mulheres e 86 homens), este autor constatou que as mulheres relatam mais do que os homens construções e pontos de referência (por exemplo, semáforo), enquanto os homens mencionam mais direções cardinais para orientação. Isto sugere que mulheres e homens diferem no tipo de referencial que utilizam para se orientar e estabelecer rotas de navegação no ambiente.

Halpern (2004) sugere que o entendimento dos processos cognitivos subjacentes às diferenças e semelhanças dos sexos permite uma análise mais refinada do que a simples comparação entre homens e mulheres em tarefas que envolvem habilidades espaciais. É neste sentido que o estilo cognitivo de sistematização é abordado no presente estudo. Coerente com esta possibilidade, ela se debruça sobre alguns processos mentais. Por exemplo, destaca que as mulheres, comparadas com os homens, apresentam acesso mais rápido a informações fonológicas, semânticas e episódicas da memória de longo prazo, e obtêm pontuações altas em testes de aprendizagem verbal e produção e compreensão de prosa complexa. Elas também apresentam vantagens em atividades quantitativas no ensino fundamental quando a aprendizagem da matemática envolve fatos e cálculo aritméticos, exigindo processo de recuperação mnemônica, similar ao que é necessário para produção e compreensão da linguagem, tendo desempenho subsequente melhor que os homens em álgebra, especificamente quando componentes cognitivos envolvidos na atividade são similares aos do processamento da linguagem. Por outro lado, os homens apresentam vantagens em teste de analogia verbal que, embora sejam verbais, envolvem relações de mapeamento na memória em termos de processamento cognitivo. Esta autora acrescenta que os homens apresentam vantagens em tarefas que requerem trabalho da memória visuo-espacial, como em testes de rotação mental ou naquelas que exigem a estimação da velocidade de objetos em movimento e compreensão do movimento em espaço tridimensional.

Em resumo, estudos prévios têm assinalado diferenças entre homens e mulheres quanto às habilidades espaciais. Entretanto, dá-se atenção mais acentuada à variável estrutural-biológica sexo do que a processos cognitivos subjacentes, a exemplo do estilo cognitivo de sistematização. Portanto, este é, provavelmente, o aspecto mais inovador do presente estudo. Além disso, procura considerar uma amostra numericamente maior do que estudos anteriores (por exemplo, Gomes \& Pinheiro, 1998; Pinheiro, 1998), incluindo pessoas da população geral. Seu objetivo principal é conhecer em que medida o sexo e o estilo de sistematização dos participantes influenciam a elaboração de elementos de mapas cognitivos no contexto brasileiro.

\section{Método}

\section{Participantes}

Participaram deste estudo 214 pessoas (sexo masculino, $43 \%, n=92$; sexo feminino, $57 \%, n=122$ ) da população geral de João Pessoa (PB), com idades variando de 18 a 60 anos ( $M$ $=29,3, D P=12,51)$. Estes tinham completado escolaridade superior $(60,3 \%)$ ou ensino médio $(39,7 \%)$. Tratou-se de uma amostra não probabilística, isto é, de conveniência, tendo participado as pessoas que, convidadas, aceitaram colaborar voluntariamente.

\section{Instrumentos}

Os participantes receberam três folhas impressas, frente e verso, contendo: (1) solicitação para listar as Unidades Federativas e respectivas capitais brasileiras; (2) quadro de dupla entrada para os respondentes estimarem as distâncias aproximadas, em quilômetros, entre João Pessoa e outras capitais das cinco regiões do Brasil (por exemplo, João Pessoa - Porto Alegre, João Pessoa - Manaus); (3) mapa mudo (esquemático) com o contorno das Unidades Federativas do Brasil para indicarem suas denominações; (4) medida de sistematização (descrita a seguir); e (5) dados demográficos (idade, sexo e escolaridade). Excetuando a última parte do questionário contendo questões sobre os dados demográficos, as demais partes foram balanceadas, minimizando o efeito de exposição.

O Quociente de Sistematização (QS) compreende uma escala originalmente elaborada em língua inglesa por Baron-Cohen e colaboradores (Baron-Cohen \& cols., 2003; Baron-Cohen \& Wheelwright, 2004), com versão em português disponibilizada (Baron-Cohen, 2004). Ela é constituída por 60 itens, sendo que 40 medem o tipo cognitivo ou de pensamento sistematizador (por 
exemplo, "O funcionamento das máquinas me fascina", "Quando olho para um quadro, não costumo pensar na técnica envolvida na pintura"). Os outros 20 itens funcionam como distratores aleatoriamente distribuídos ao longo do questionário para distrair o participante quanto ao foco na sistematização e não são incluídos no escore final (por exemplo, "Gosto de praticar esportes"). Os respondentes indicam suas respostas em escala tipo Likert, de quatro pontos, variando de 1 (Não me descreve nada) a 4 (Descreve-me totalmente).

\section{Procedimentos}

Conforme a resolução 196/96, do Conselho Nacional de Saúde, esta pesquisa respeitou a autonomia do indivíduo, a beneficência, a não maleficência, a justiça e equidade. Para a realização da pesquisa foram considerados também os princípios éticos e o código de conduta promovidos pela American Psychological Association [APA] (2002).

A coleta de dados foi realizada individualmente. Cada pessoa era abordada em sua residência ou local de trabalho e solicitada a colaborar com a pesquisa, informando que sua participação seria voluntária e anônima. Indicou-se que não era o propósito testar seu conhecimento, mas ter informações sobre como as pessoas localizam cidades e estados brasileiros, além de saber a forma como elas costumam pensar e agir no seu dia a dia. A todos foi informado que ao aceitar participar no estudo, estariam dando seu consentimento livre e esclarecido para que os dados fossem tratados no seu conjunto.

Uma equipe de dez colaboradores previamente treinados fez a coleta dos dados. Em média, 25 minutos foram demandados para concluir a participação no estudo.

\section{Análise de dados}

Os dados foram analisados com o SPSS (versão 18). Além de estatísticas descritivas (média, desvio padrão, frequência, intervalo de confiança e erro padrão), foram utilizados análise de componentes principais, análise de regressão e testes de comparação de médias ( $t$ de Student). Estes últimos foram realizados para saber se homens e mulheres se diferenciavam em termos de sistematização, acertos, omissões e erros ao listar as Unidades Federativas, suas capitais e localizações no mapa mudo.

Para estimar as distâncias aproximadas em quilômetros, entre João Pessoa e capitais das cinco regiões do Brasil, realizaram-se Escalonamentos Multidimensionais (MDS) visando conhecer as configurações dos mapas cognitivos de homens e mulheres em termos de distribuição dos estados, tendo como referência as dimensões Norte - Sul e Leste - Oeste. Ou seja, teve-se como objetivo conhecer como homens e mulheres representam o mapa do Brasil em termos cognitivos, acessando tal informação por meio de "método cartográfico", no qual se objetiva reproduzir a "geografia" do ambiente em termos de mapas cognitivos. No caso, o $M D S$ permitiu construir uma configuração espacial a partir de juízos subjetivos de distâncias que presumivelmente representam a informação armazenada pelos indivíduos sobre a localização de uma série de elementos do ambiente, e que se pode contrastar com mapas reais (Ruiz $\&$ Valiñas, 1991).

\section{Resultados}

Inicialmente, procurou-se criar uma pontuação total de sistematização, realizando uma análise de componentes principais com os itens do $Q S[K M O=0,74$, Teste de Esfericidade de Bartlett, $\left.\chi^{2}(780)=2.143,20, p<0,001\right]$. O componente geral observado apresentou valor próprio (eigenvalue) de 5,73, explicando $14,3 \%$ da variância total. Considerando o critério de carga fatorial igual ou superior a 0,40 , foram selecionados 16 itens. Portanto, estes itens são os que melhor representam sistematização para a presente amostra. Os itens foram somados para criar uma pontuação total, que quanto maior mais a pessoa é considerada sistematizadora. Esta versão abreviada post hoc será considerada a seguir, tendo apresentado consistência interna (Alfa de Cronbach) de $0,83^{1}$.

As estatísticas descritivas das variáveis de interesse são descritas na Tabela 1. Como é possível observar nesta tabela, os homens apresentaram maior média que as mulheres para as três variáveis de interesse: número de pares corretos UFcapital, número de localizações corretas no mapa esquemático e pontuação média em sistematização.

Estes resultados ficam evidentes ao observar os diagramas de barras de erro da Figura 1. Entretanto, observa-se que na Figura 1(b), entre as linhas tracejadas, encontra-se a área de sobreposição de uma variável sobre a outra. Isso sugere que, de fato, pode não existir uma diferença real entre os homens e mulheres em termos de localização das UFs, pois as médias populacionais podem ser as mesmas. O mesmo não ocorreu para as demais variáveis, pois não houve sobreposição dos ICs de $95 \%$. Portanto, constatou-se uma diferença real entre homens e mulheres quanto ao número de pares corretos UF-capital e pontuação média em sistematização. Isso indica que as médias populacionais entre homens e mulheres para estas duas variáveis são "muito provavelmente" diferentes. "Muito provavelmente", pois este julgamento se baseia em probabilidades, deve-se assumir o risco, a margem de erro, dependente de fatores casuais, pouco prováveis, mas possíveis.

Contudo, esta representação gráfica não é suficiente para identificar diferenças e semelhanças entre homens e mulheres. Neste sentido, compararam-se as pontuações médias de homens e mulheres por meio do teste $t$. A diferença de médias em relação ao número de pares corretos UF-capital foi de 3,27 (IC de 95\% de $1,37$ a 5,18$)$, com tamanho de efeito mediano $(d=0,46)$. [Cohen (1992) recomenda que para o tamanho do efeito de teste $t, 0,20$ é pequeno, 0,50 médio e 0,80 grande]. O teste $t[(212)=3,39$, $p=0,01]$ indicou diferença entre homens e mulheres quanto à lembrança destes pares.

No que concerne ao número de localizações corretas no mapa esquemático, o teste $t[(212)=1,87, p=0,063]$ indicou que não houve diferença significativa nas pontuações médias, embora os participantes do sexo masculino tenham apresentado um valor um pouco maior (ver Tabela 1). A diferença de médias entre homens e mulheres foi de 2,10 (IC de 95\% de 0,11 a 4,32).

Com relação à pontuação em sistematização, a diferença de médias entre homens e mulheres foi de 7,54 (IC de 95\% = 5,45$9,63)$, com tamanho de efeito grande $(d=0,99)$. O teste $t[(212)$ $=7,12, p=0,000]$ revelou que a diferença foi estatisticamente 
Tabela 1

Média, desvio padrão, erro padrão e IC de 95\% para homens e mulheres

\begin{tabular}{|c|c|c|c|c|c|c|}
\hline & \multicolumn{2}{|c|}{$\begin{array}{l}\text { Número de pares corretos } \\
\text { UF-Capital }\end{array}$} & \multicolumn{2}{|c|}{$\begin{array}{l}\text { Número de localizações } \\
\text { corretas no mapa } \\
\text { esquemático }\end{array}$} & \multicolumn{2}{|c|}{$\begin{array}{l}\text { Pontuação } \\
\text { sistematização }\end{array}$} \\
\hline & $\mathrm{F}$ & $\mathrm{M}$ & $\mathrm{F}$ & M & $\mathrm{F}$ & $\mathrm{M}$ \\
\hline Média & 14,0 & 17,2 & 16,8 & 18,9 & 31,7 & 39,3 \\
\hline Desvio padrão & 6,65 & 7,44 & 8,27 & 7,97 & 7,90 & 7,34 \\
\hline Erro padrão & 0,60 & 0,78 & 0,75 & 0,83 & 0,72 & 0,77 \\
\hline IC de $95 \%$ & $12,8-15,2$ & $15,7-18,8$ & $15,3-18,3$ & $17,2-20,5$ & $30,3-33,1$ & $37,7-40,8$ \\
\hline
\end{tabular}

Legenda. $\mathrm{F}=$ Feminino $(n=122), \mathrm{M}=$ Masculino $(n=92), \mathrm{IC}=$ intervalo de confiança
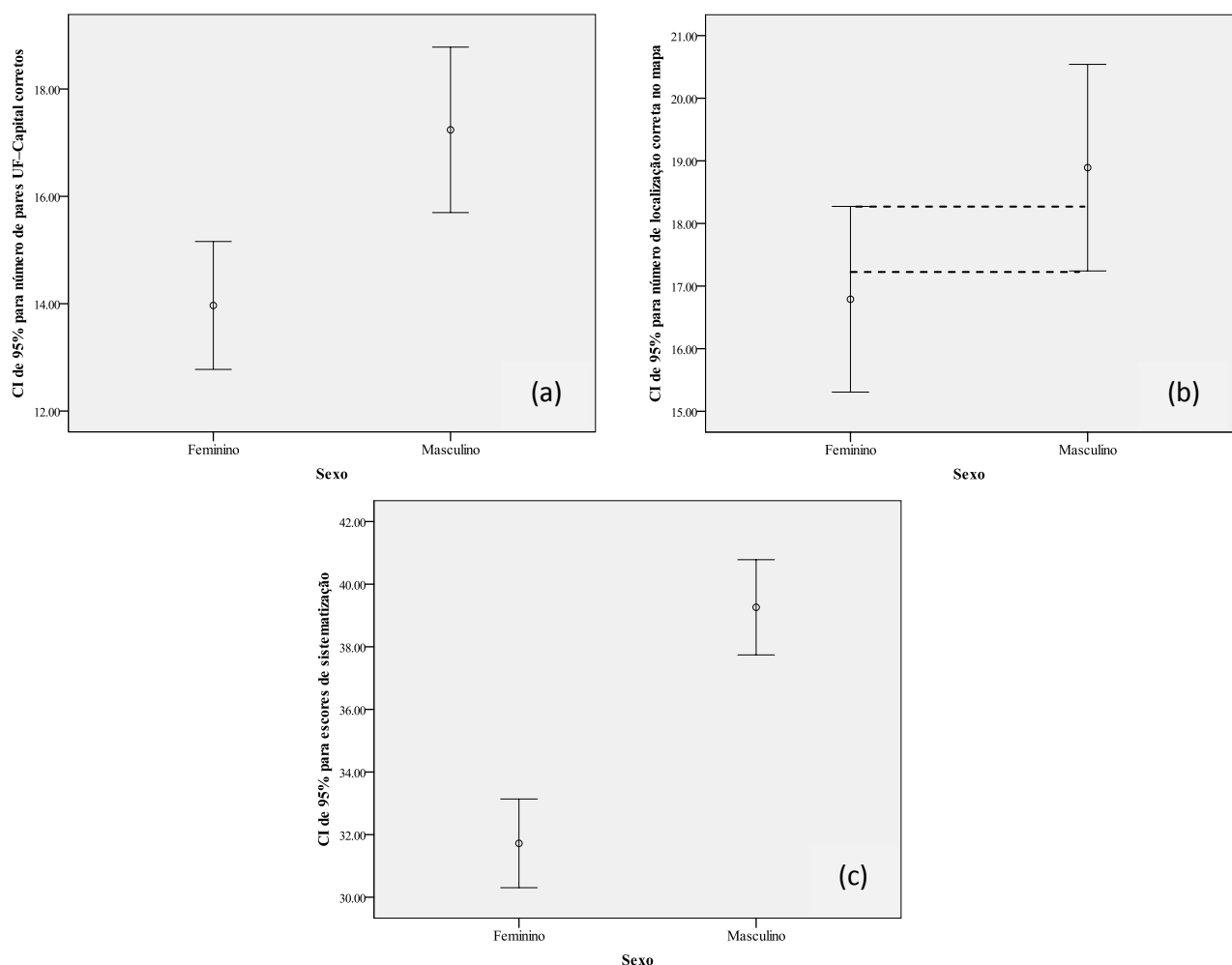

Figura 1. Barras de erro por sexo para (a) pares corretos UF-Capital, (b) localizações corretas no mapa e (c) pontuação em sistematização.

significativa, indicando que ser do sexo masculino ou feminino determina padrões diferentes de sistematização. Neste caso, verificou-se que os homens apresentaram maior pontuação média em sistematização do que as mulheres.

Para conhecer como homens e mulheres representam o mapa do Brasil em termos cognitivos, efetuou-se uma $M D S$ para os homens [Figura 2(a); $R S Q=0,99$ e $S$-stress $=0,07]$ e outra para as mulheres [Figura 2(b); $R S Q=0,97$ e $S$-stress $=$ $0,12]$. Comparando as duas figuras, percebe-se que, tendo como referência as direções cardinais Norte-Sul e Leste-Oeste, as capitais dos estados estão melhor distribuídas na Figura 2(a) dos homens do que na Figura 2(b) das mulheres.

Finalmente, buscou-se estimar o efeito independente das variáveis sexo e sistematização sobre as duas variáveis de mapas cognitivos. Para tal fim realizou-se regressão linear do tipo stepwise para examinar qual das variáveis independentes (sexo e/ou sistematização) permaneceria na equação para explicar os aspectos de mapas cognitivos aqui tratados. As correlações entre as variáveis são apresentadas na Tabela 2. Explicando o número de pares corretos UF-Capital, sexo foi um preditor significante $(\beta$ $=.23, p<.01)$, tendo sido a variável sistematização excluída da equação. Inversamente, para o número de localizações corretas no mapa esquemático, sistematização foi um preditor significante $(\beta=.21, p<.01)$, mas a variável sexo foi excluída da equação. Estes resultados indicam efeito de supressão nas equações de regressão e que, embora relacionados, sexo e estilo cognitivo de sistematização apresentam efeitos independentes para variáveis específicas de mapas cognitivos.

\section{Discussão}

O objetivo deste estudo foi examinar em que medida homens e mulheres se diferenciam quanto ao estilo cognitivo de sistematização e à habilidade de construção de mapas cognitivos. 

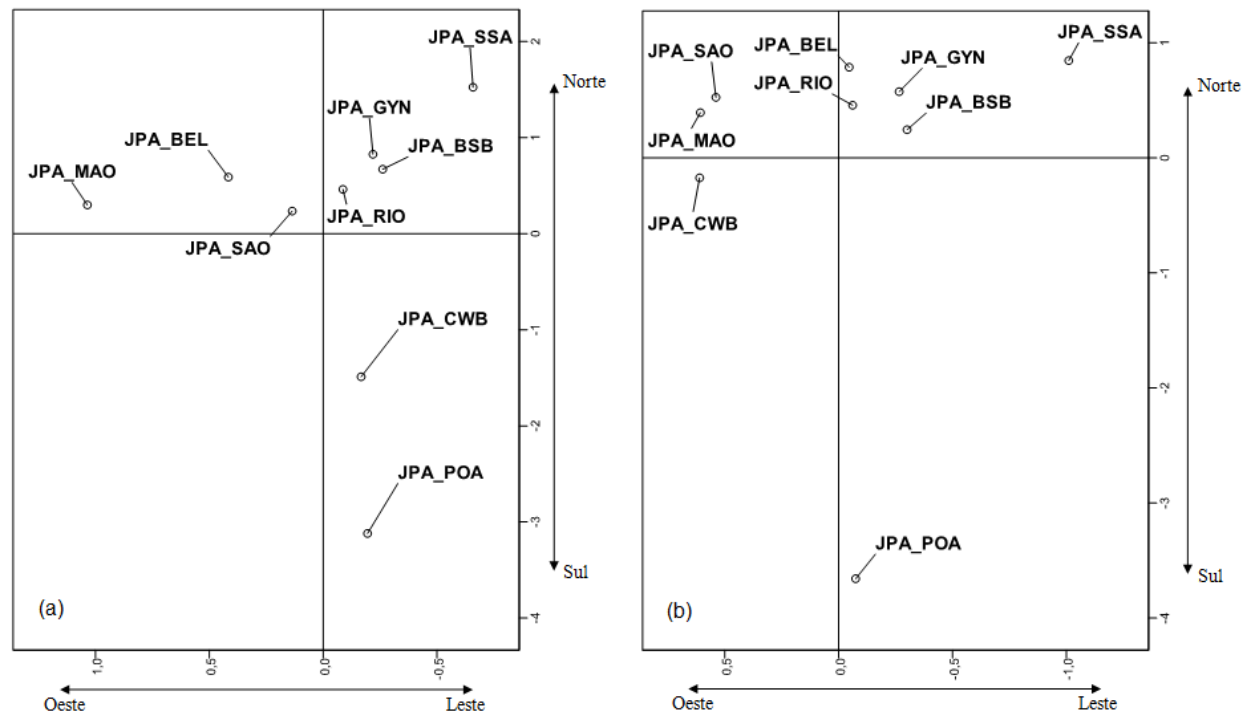

Figura 2. MDS para distâncias estimadas entre capitais por pessoas (a) do sexo masculino e (b) do sexo feminino JPA-SSA = João Pessoa/Salvador; JPA-MAO = João Pessoa/Manaus; JPA-BEL = João Pessoa/Belém; JPA$\mathrm{SAO}=$ João Pessoa/São Paulo; JPA-RIO = João Pessoa/Rio de Janeiro; JPA-GYN = João Pessoa/Goiânia; JPABSB = João Pessoa/BSB; JPA-CWB = João Pessoa/Curitiba; JPA-POA = João Pessoa/Porto Alegre.

Tabela 2

Correlações entre as variáveis de interesse

\begin{tabular}{llll}
\hline Variáveis & 1 & 2 & 3 \\
\hline 1. Sexo & & & \\
2. Pares corretos UF-Capital & $0,23^{* *}$ & & \\
3. Localizações corretas & $0,13^{*}$ & $0,56^{* * *}$ & \\
4. Sistematização & $0,44^{* * *}$ & $0,20^{* *}$ & $0,21^{* *}$ \\
\hline
\end{tabular}

Legenda. $* * * p<0,001 . * * p<0,01 .{ }^{\dagger} p<0,07$.

Os resultados indicaram que (comparado às participantes mulheres), os participantes homens (1) têm mais facilidade para lembrar e listar as Unidades Federativas (UFs) e respectivas capitais brasileiras, (2) têm mais facilidade para localizar corretamente as UFs em um mapa esquemático do Brasil (embora esta diferença não tenha sido estatisticamente significativa) e (3) têm um estilo cognitivo de sitematização maior. Estes três resultados principais corroboram estudos prévios e são discutidos em mais detalhe abaixo.

Os homens se lembraram de mais pares corretos UF-capital do que as mulheres. Este resultado parece congruente com o fato de que os homens apresentam vantagens em teste de analogia verbal (Halpern, 2004). Como discutido na introdução, a tarefa é verbal, mas em termos de processamento cognitivo, envolve relações de mapeamento. Neste sentido, pode-se pensar que lembrar as UFs e suas respectivas capitais compreenda uma tarefa que envolve relações de mapeamento na memória. Portanto, em média, nestas condições os homens apresentaram maior número de acertos do que as mulheres. A análise adicional de regressão indicou que é exatamente a variável sexo que tem maior importância com respeito à tarefa de lembrar pares corretos UF-capital. Ou seja, embora também sistematizem mais do que as mulheres (ver discussão abaixo), o fato de os homens se lembrarem de mais pares UF-capital corretos parece independer neste caso de suas pontuações em sistematização.

Os homens também tiveram mais facilidade para localizar corretamente as UFs em um mapa esquemático do Brasil, porém esta diferença não foi estatisticamente significante. $\mathrm{Ou}$ seja, a diferença encontrada não é suficientemente grande para supor que homens e mulheres de fato difiram no que se refere à localização espacial das UFs brasileiras. Cabe especular que isso pode dever-se à dimensão continental do Brasil, fracionado em 27 UFs; talvez fosse o caso de no futuro tomar como referência a região ou o estado dos participantes, pedindo para que localizem unidades geográficas específicas. Foi citado anteriormente o estudo de Montello e cols. (1999) que indica que, em atividades que envolvem a recordação de objeto-localização, as mulheres apresentam menos erros. É possível que este aspecto tenha contribuído para a diferença pequena entre os sexos relatada no presente estudo com relação a localizar corretamente as UFs. Chama a atenção que, embora alguns estudos valorizem as diferenças a respeito, estas não são gerais em termos de habilidades cognitivas espaciais, sobretudo em mapas cognitivos. Deste modo, constata-se que existem diferenças pontuais, isto é, em atividades específicas, principalmente quando bem definidas as tarefas que envolvem aspectos visuo-espaciais e rotação mental, mais característicos do cérebro masculino. Esta situação demanda maior gradiente de sistematização do que tarefas que envolvem conhecimento verbal, em que o melhor desempenho é dado por pessoas do sexo feminino (Baron-Cohen, 2002).

Destaca-se ainda que a tarefa de localizar as UFs no mapa do Brasil com os contornos dos estados pode envolver conhecimento 
verbal, tratando-se também de conhecimento de Geografia que pode ter sido adquirido na escola, não necessariamente a partir de experiências ou vivências no próprio ambiente, potencialmente influenciando o resultado previamente descrito. Pode-se conjeturar que, caso não existissem os contornos e a atividade requerida envolvesse conhecimento adquirido direto no ambiente (por exemplo, localização de lugares no mapa do bairro, cidade), talvez houvesse diferença maior entre homens e mulheres, pois trataria de processos cognitivos que demandariam transformar informações 3-D em 2-D, ambiente em mapa, pois como indica Baron-Cohen (2002), este procedimento implica em necessidade de sistematização. A análise adicional de regressão mostrou que a sistematização é a variável com maior importância relacional com a tarefa de localizar UFs. Portanto, embora haja certa relação com a variável sexo, localizar UFs em mapa mudo parece depender mais de pontuações em sistematização.

Esta suposição de que a tarefa envolve conhecimento geográfico verbal, e daí a explicação para pequena diferença, é coerente com os achados de Gomes e Pinheiro (1998) sobre o desempenho de homens e mulheres quanto à exteriorização da representação interna do mapa cognitivo do mundo. Estes autores relatam que "homens e mulheres tiveram basicamente o mesmo desempenho quando o fator considerado foi o 'nome' do país; ou seja, os nomes dos países foram guardados na memória, e resgatados de lá, de modo similar por ambos os grupos" (p. 143). Deste modo, estes achados e os descritos aqui vão na mesma direção, ratificando a interpretação de que o conhecimento geográfico verbal não tende a apresentar diferenças em função dos sexos.

Verificou-se também que os homens apresentaram maior pontuação média em sistematização do que as mulheres. Este achado é consistente com a teoria e as pesquisas na área (BaronCohen \& cols, 2002; Baron-Cohen \& cols., 2003; Billington et al.,2007; Wheelwright \& cols., 2006). O cérebro masculino é mais sistemático, explicando que os homens tenham melhor desempenho em atividades cognitivas visuo-espaciais ou que envolvem processos de sistematização (Baron-Cohen, 2002).

Neste sentido, estima-se que diferenças de sexo em atividades como estimar distâncias tem como fator subjacente um processo cognitivo, tratado aqui como a dimensão psicológica de sistematização. Como desmonstrado na Figura 2, os homens representam o mapa do Brasil em termos cognitivos mais eficazmente (isto é, as capitais dos estados estão mais bem distribuídas) do que as mulheres. Este resultado corrobora os já encontrados em outros estudos e dá sustentação para a suposição teórica de que pessoas do sexo masculino apresentam vantagens em visualizar objetos em rotação e calcular distâncias, podendo ter como determinante a dimensão psicológica sistematização (Baron-Cohen, 2002).

\section{Considerações Finais}

Neste estudo foram considerados dois tipos de variáveis que, ao menos epistemologicamente, têm naturezas diversas. Por um lado, incluiu-se a variável sexo, que tem uma natureza estritamente biológica, embora no contexto social e no mundo cotidiano reflita-se em papéis de gênero, reservando aos homens atividades mais pragmáticas, vinculadas com questões sistemáticas, enquanto às mulheres atividades mais humanas e relacionais. Por outro lado, existe a variável sistematização que, como definida por Baron-Cohen (2002), tem natureza mais ontogenética. Estima-se que homens e mulheres nascem com alguma predisposição a pensar de forma sistematizadora e empatizadora, respectivamente. Embora o presente estudo junte-se a outros ao demonstrar que homens tendem a apresentar maior sistematização do que as mulheres, não se pode perder de vista que esta diferenciação também foi observada no presente estudo. A variável sexo e o estilo cognitivo de sistematização explicaram diferentemente aspectos de mapas cognitivos.

Portanto, os resultados do presente estudo têm implicações teóricas e práticas. No âmbito teórico, considerar diferenças e semelhanças em relação ao sexo, levando em conta o estilo cognitivo de sistematização, é uma forma de compreender os aspectos subjacentes ao funcionamento cognitivo de homens e mulheres, mais especificamente em relação à construção de mapas cognitivos. No caso, constatou-se que os homens apresentam vantagens quando a tarefa envolve aspectos visuoespaciais, como estimação de distâncias, que demanda maior gradiente de sistematização; esta tendência é mais evidente do que quando as tarefas envolvem conhecimento geográfico verbal, em que as diferenças entre os sexos são menores.

No âmbito prático, no que diz respeito ao ensino da Geografia, os educadores devem ter consciência de que pessoas dos sexos masculino e feminino apresentam habilidades diferentes para interpretar ou utilizar informações espaciais ou conhecimentos geográficos. Isso demanda empregar estratégias diferentes de ensino em sala de aula, procurando tratar com alguma diferença homens e mulheres. Não se pretende eliminar diferenças de sexo, mesmo porque estas tendem a ser estruturais e biológicas, como refletidas nos cérebros masculino (sistemático) e feminino (empático) (Baron-Cohen, 2004); mas sim incentivar modos diferentes de ensino-aprendizagem em razão do sexo.

Finalmente, devem-se ponderar os resultados aqui apresentados, considerando a necessidade de replicar a pesquisa. Neste caso, talvez valesse a pena pensar em espaços geoespaciais mais próximos da realidade das pessoas, como os representados pelas cidades e bairros em que vivem. Existem evidências de que as pessoas avaliam riscos ambientais diferentemente quando estes são apresentados em relação a espaços geoespaciais mais próximos ou mais distantes, tendendo, por exemplo, a avaliar riscos ambientais mais distantes geograficamente como mais severos do que os mais próximos (Milfont, Abrahamse, \& McCarthy, 2011). Talvez proximidade e distância em espaços geoespaciais influenciem a representação de mapas cognitivos. Quem sabe fosse igualmente interessante levantar informações sobre como a pessoa elaborou seu conhecimento acerca do ambiente, se por meio de aprendizagem formal ou vivências pessoais.

\section{Referências}

American Psychological Association (2002). Ethical Principles of Psychologists and code of conduct. American Psychologist, 57, 1060-1073

Aragonés, J. I. (1991). Cognición ambiental. In F. J. Burillo \& J. I. Aragonés 
(Orgs.), Introducción a la psicología ambiental (pp. 65-81). Madrid: Alianza Editorial.

Baron-Cohen, S. (2002). The extreme male brain theory of autism. Trends in Cognitive Sciences, 6, 248-254.

Baron-Cohen, S. (2004). Diferença essencial: A verdade sobre o cérebro de homens e mulheres. Rio de Janeiro: Objetiva.

Baron-Cohen, S., Richler, J., Bisarya, D., Gurunathan, N., \& Wheelwright, S. (2003). The systemizing quotient: an investigation of adults with Asperger syndrome or high-functioning autism, and normal sex differences. Philosophical Transactions of the Royal Society of London: Series B-Biological Sciences, 358, 361-374.

Baron-Cohen, S. \& Wheelwright, S. (2004). The empathy quotient: an investigation of adults with Asperger syndrome or high functioning autism, and normal sex differences. Journal of Autism and Developmental Disorders, 34, 163-175.

Billington, J., Baron-Cohen, S. \& Whweelwright, S. (2007). Cognitive style predicts entry into physical sciences and humanities: questionnaire and performance tests of empathy and systemizing. Learning and Individual Differences, 17, 260-268.

Cohen, J. (1992). A power prime. Psychological Bulletin, 112, 155-159.

Downs, R. M. \& Stea, D. (1973). Cognitive maps and spatial behaviour: process and products. In R. M. Downs \& D. Stea (Orgs.), Image and environment (pp. 8-26). Chicago: Aldine.

Focquaert, F., Steven, M. S., Wolford, G. L., Colden, A., \& Gazzaniga, M. S. (2007). Empathizing and systemizing cognitive traits in the sciences and humanities. Personality and Individual Differences, 43, 619-625.

Golledge, R. G. (2002). The open door of GIS. In R. B. Bechtel \& A. Churchman (Orgs.), Handbook of environmental psychology (pp. 244-255). Nova Jersey: John Wiley \& Sons.

Gomes, M. V. S., \& Pinheiro, J. Q. (1998). Influência do gênero em mapas cognitivos do mundo de universitários brasileiros. Estudos de Psicologia, 3, 139-146.

Gouveia, V. V., Milfont, T. L., Gouveia, R. S. V., Rique, J., \& Galvao, L. (no prelo). Short form of the empathy quotient (EQ-15): evidences of its construct validity and reliability. Spanish Journal of Psychology.

Halpern, D. F. (2004). A cognitive-process taxonomy for sex differences in cognitive abilities. American Psychological Society, 13, 135-139.

Lawton, C. A. (2001). Gender and regional differences in spatial referents used in direction giving. Sex Role, 44, 321-337.

Kitchin, R. M. (1994). Cognitive maps: what are they and why study them? Journal of Environmental Psychology, 14, 1-19.

Lawrence, E. J., Shaw, P., Baker, D., Baron-Cohen, S. \& David, A. S. (2004) Measuring empathy: reliabitity and validity of the Empathy Quotient. Psychological Medicine, 34, 911-924.

Milfont, T. L., Abrahamse, W., \& McCarthy, N. (2011). Spatial and temporal biases in assessments of environmental conditions in New Zealand. New Zealand Journal of Psychology, 40, 56-67.

Montello, D. R., Lovelace, K. L., Reginald, G. G., \& Self, C. M. (1999). Sexrelated differences and similarities in geographic and environmental spatial abilities. Annals of the Association of American Geographers, 89, 515-534.

Moser, G., \& Uzzell, D. (2003). Environmental psychology. In T. Millon \& M. J. Lerner (Orgs.), Comprehensive handbook of psychology (vol. 5, pp. 419445). Nova Iorque: John Wiley \& Sons.

Nettle, D. (2007). Empathizing and systemizing: what are they, and what do they contribute to our understanding of psychological sex differences? British Journal of Psychology, 98, 237-255.

Pinheiro, J. Q. (1998). Determinants of cognitive maps of the world as expressed in sketch maps. Journal of Environmental Psychology, 18, 321-339.

Ruiz, H. B. \& Valiñas, C. M. (1991). Metodos de investiogacion de mapas cognitivos. In J. Burillo \& J. I. Aragonés (Orgs.), Introducción a la psicología ambiental (pp. 83-93). Madrid: Alianza Psicología.

Wheelwright, S., Baron-Cohen, S., Goldenfeld, N., Delaney, J., Fine, D., Smith, R., ...Wakabayashi, A. (2006). Predicting Autism Spectrum Quotient (AQ) from the Systemizing Quotient-Revised (SQ-R) and Empathy Quotient (EQ). Brain Research, 1079, 47-56.

1. Os itens da escala selecionados foram: $5,7,13,15,18,20,29,33,34,37,40,41,48,49,53$ e 55. Homens e mulheres também apresentam diferenças significativas quando é utilizada a pontuação de sistematização com todos os itens. A versão abreviada post hoc foi preferida, pois representa os melhores itens e o núcleo principal de sistematização. Esta versão com 16 itens apresentou correlação alta $(r=0,44)$ com a pontuação formada pelos outros 24 itens.

Taciano Lemos Milfont, doutor em Psicologia Social e Ambiental pela Universidade de Auckland e pósdoutor em Psicologia Ambiental pela California State University, é professor e membro do Centre for Applied Cross-Cultural Research da Victoria - University of Wellington. Endereço para correspondência: School of Psychology, Victoria University of Wellington,

PO Box 600, Wellington, New Zealand, 6140. Fone: +64-4-463 6398, Fax: +64-4-463 5402. E-mail:Taciano. Milfont@vuw.ac.nz

Jorge Artur Peçanha de Miranda Coelho, doutor em Psicologia Social pela Universidade Federal da Paraíba, é professor Adjunto II da Universidade Federal de Alagoas. E-mail: orgearturcoelho@pesquisador.cnpq.br

Viviany Silva Pessoa, doutora em Psicologia Social pela Universidade Federal da Paraíba (UFPB).

Valdiney Veloso Gouveia, doutor em Psicologia Social pela Universidade Complutense de Madri, é professor Titular na Universidade Federal da Paraíba. E-mail: vvgouveia@gmail.com 